\title{
Yet another generalization of frames and Riesz bases
}

\author{
Reza Joveini and Massoud Amini \\ (Communicated by David Larson)
}

\begin{abstract}
A frame is a sequence of vectors in a Hilbert space satisfying certain inequalities that make it valuable for signal processing and other purposes. There is a formula giving the reconstruction of a signal (a vector in the space) from its sequence of inner products (the Fourier coefficients) with the elements of the frame sequence. A $g$-frame, or operator-valued frame, is a sequence of operators defined on a countable ordered index set that has properties analogous to those of a frame sequence.

We present a new approach to the matter of defining a Hilbert space frame, indexed by an ordered set, when the set is a measure space which is not necessarily purely atomic. Continuous frames have been widely studied in the literature, but the measure spaces they are associated with are not necessarily ordered in any way. Our approach is to make the measure space a directed set, and then replace the sequence of vectors (or operators) with a net indexed by the directed set, obtaining a natural generalization of the usual notion of generalized frame. We show that this definition makes sense mathematically, and proceed to obtain generalizations of several of the standard results for frame and Bessel sequences, and also Riesz bases, $g$-frames and operator-valued frames.
\end{abstract}

\section{Introduction}

Frames are generalizations of bases in a Hilbert space. They were introduced and studied in [Duffin and Schaeffer 1952] and [Daubechies et al. 1986]. They have been recently of special interest because of their applications in signal processing. The interested reader is referred to [Christensen 2003; Daubechies 1992; Feichtinger and Strohmer 1998; Gröchenig 2001; Han and Larson 2000; Heil and Walnut 1989; Yong 1980] for theory and applications of frames. Throughout this paper, $U$ and $V$ are two Hilbert spaces and $\left\{V_{m}: m \in M\right\}$ is a net of subspaces of

MSC2000: primary 42C15, 42C99; secondary 42C40.

Keywords: $g$-frames, $g$-Riesz bases.

This is part of the first author's M. Sc. Thesis at Tarbiat Modares University. 
$V, \mathscr{B}\left(U, V_{m}\right)$ is the collection of all bounded linear operators from $U$ into $V_{m}$ and $(M, \mu)$ is a measure space.

Definition 1.1. We call a net $\left\{\Lambda_{m} \in \mathscr{B}\left(U, V_{m}\right): m \in M\right\}$ a generalized frame or simply a $g$-frame for $U$ with respect to $\left\{V_{m}: m \in M\right\}$ if

(a) for each $f \in U$, there is a measurable function $\tilde{f}: M \rightarrow V_{m}$ such that $\tilde{f}(m)=$ $\Lambda_{m} f$, and

(b) there are positive constants $A$ and $B$ such that

$$
A\|f\|_{U}^{2} \leq\|\tilde{f}\|_{L_{\left(M, V_{m}\right)}^{2}}^{2} \leq B\|f\|_{U}^{2} \quad(f \in U) .
$$

We call $A$ and $B$ the lower and upper frame bounds. We call $\left\{\Lambda_{m}: m \in M\right\}$

(i) a tight $g$-frame if $A=B$;

(ii) an exact $g$-frame if it ceases to be a $g$-frame whenever any of its elements is removed;

(iii) a $g$-frame for $U$ whenever the net $\left\{V_{m}: m \in M\right\}$ is clear from the context;

(iv) a $g$-frame for $U$ with respect to $V$ whenever $V_{m}=V$, for each $m \in M$.

Various generalizations of frames have been proposed [Aldroubi et al. 2004; Asgari and Khosravi 2005; Casazza and Kutyniok 2004; Christensen and Eldar 2004; Feichtinger and Strohmer 1998; Fornasier 2003; Gröchenig 2001]. We take as our starting point the generalization presented in [Sun 2006]. Our definition above is just the definition of $g$-frames in [Sun 2006] when the measure space $M$ is countable, $\mu$ is the counting measure and $V_{m}=\mathbb{C}$ for $m \in M$. The case when $M$ is not countable could be of interest when one deals with nonseparable Hilbert spaces (such as Hilbert space completions of the space of almost periodic functions). Thus our present work can be regarded as a nonseparable version of [Sun 2006]. For instance Examples 3.4 and 3.5 in that reference are outside the scope of Sun's definition when the Hilbert space has no countable Riesz basis, but they fit in our framework.

\section{2. $g$-frame operators and dual $g$-frames}

Let $\left\{\Lambda_{m}: m \in M\right\}$ be a $g$-frame for $U$ with respect to $\left\{V_{m}: m \in M\right\}$. Define the $g$-frame operator $S$ by

$$
S f=\int_{M} \Lambda_{m}^{*} \Lambda_{m} f d \mu(m),
$$

where $\Lambda^{*}$ is the adjoint operator of $\Lambda$. 
Lemma 2.1. Let $(\Omega, \mu)$ be a measure space, $X$ and $Y$ are two Banach spaces, $\lambda: X \rightarrow Y$ be a bounded linear operator and $f: \Omega \rightarrow X$ be a measurable function. Then

$$
\lambda\left(\int_{\Omega} f d \mu\right)=\int_{\Omega}(\lambda f) d \mu .
$$

Next we want to show $S$ is self-adjoint operator. For any $f_{1}, f_{2} \in U$ we have

$$
\begin{aligned}
\left\langle S f_{1}, f_{2}\right\rangle & =\left\langle\int_{M} \Lambda_{m}^{*} \Lambda_{m} f_{1} d \mu(m), f_{2}\right\rangle=\int_{M}\left\langle\Lambda_{m}^{*} \Lambda_{m} f_{1}, f_{2}\right\rangle d \mu(m) \\
& =\int_{M}\left\langle f_{1}, \Lambda_{m}^{*} \Lambda_{m} f_{2}\right\rangle d \mu(m)=\left\langle f_{1}, \int_{M} \Lambda_{m}^{*} \Lambda_{m} f_{2} d \mu(m)\right\rangle=\left\langle f_{1}, S f_{2}\right\rangle .
\end{aligned}
$$

Moreover, $S$ is a bounded operator because

$$
\begin{aligned}
\|S\| & =\sup _{\|f\|=1}\|S f\|=\sup _{\|f\|=1}\left\|\int_{M} \Lambda_{m}^{*} \Lambda_{m} f d \mu(m)\right\| \leq \sup _{\|f\|=1}\left(\int_{M}\left\|\Lambda_{m}^{*} \Lambda_{m} f\right\| d \mu(m)\right) \\
& =\sup _{\|f\|=1} \int_{M}\left(\left\langle\Lambda_{m} f, \Lambda_{m} f\right\rangle\right)^{1 / 2} d \mu(m)=\sup _{\|f\|=1} \int_{M}\|\tilde{f}(m)\| d \mu(m) \leq B .
\end{aligned}
$$

Since $A\|f\|^{2} \leq\langle S f, f\rangle \leq\|S f\|\|f\|$, we have

$$
\|S f\| \geq A\|f\|,
$$

which implies that $S$ is injective and $S U$ is closed in $U$. Let $f_{2} \in U$ be such that $\left\langle f_{1}, S f_{2}\right\rangle=0$, for each $f_{1} \in U$. This implies that $S f_{2}=0$ and therefore $f_{2}=0$. Hence $S U=U$. Consequently $S$ is invertible and $\left\|S^{-1}\right\| \leq \frac{1}{A}$. For any $f \in U$ we have

$$
f=S S^{-1} f=S^{-1} S f=\int_{M} \Lambda_{m}^{*} \Lambda_{m} S^{-1} f d \mu(m)=\int_{M} S^{-1} \Lambda_{m}^{*} \Lambda_{m} f d \mu(m) .
$$

Let $\tilde{\Lambda}_{m}=\Lambda_{m} S^{-1}$. Then the above equalities become

$$
f=\int_{M} \Lambda_{m}^{*} \tilde{\Lambda}_{m} f d \mu(m)=\int_{M} \tilde{\Lambda}_{m}^{*} \Lambda_{m} f d \mu(m) .
$$

We now prove that $\left\{\tilde{\Lambda}_{m}: m \in M\right\}$ is also a $g$-frame for $U$ with respect to the set $\left\{V_{m}: m \in M\right\}$; in fact for any $f \in U$ we have

$$
\begin{aligned}
\int_{M}\left\|\tilde{\Lambda}_{m} f\right\|^{2} d \mu & =\int_{M}\left\|\Lambda_{m} S^{-1} f\right\|^{2} d \mu=\int_{m}\left\langle\Lambda_{m} S^{-1} f, \Lambda_{m} S^{-1} f\right\rangle d \mu \\
& =\int_{M}\left\langle\Lambda_{m}^{*} \Lambda_{m} S^{-1} f, S^{-1} f\right\rangle d \mu \\
& =\left\langle S S^{-1} f, S^{-1} f\right\rangle=\left\langle f, S^{-1} f\right\rangle \leq \frac{1}{A}\|f\|^{2} .
\end{aligned}
$$


On the other hand, since

$$
\begin{aligned}
\|f\|^{2} & =\int_{M}\left\langle\tilde{\Lambda}_{m}^{*} \Lambda_{m} f, f\right\rangle d \mu=\int_{M}\left\langle\Lambda_{m} f, \tilde{\Lambda}_{m} f\right\rangle d \mu \\
& \leq\left(\int_{M}\left\|\Lambda_{m} f\right\|^{2} d \mu\right)^{1 / 2}\left(\int_{M}\left\|\tilde{\Lambda}_{m} f\right\|^{2} d \mu\right)^{1 / 2} \leq B^{1 / 2}\|f\|\left(\int_{M}\left\|\tilde{\Lambda}_{m} f\right\|^{2} d \mu\right)^{1 / 2},
\end{aligned}
$$

we have

$$
\int_{M}\left\|\tilde{\Lambda}_{m} f\right\|^{2} d \mu \geq \frac{1}{B}\|f\|^{2} .
$$

Hence, $\left\{\tilde{\Lambda}_{m}: m \in M\right\}$ is a $g$-frame for $U$ with frame bounds $A^{-1}$ and $B^{-1}$. We call it the (canonical) dual $g$-frame of $\left\{V_{m}: m \in M\right\}$.

Let $\tilde{S}$ be the $g$-frame operator associated with $\left\{\tilde{\Lambda}_{m}: m \in M\right\}$. Then, for $f \in U$,

$$
\begin{aligned}
S \tilde{S} f & =\int_{M} S \tilde{\Lambda}_{m}^{*} \tilde{\Lambda}_{m} f d \mu=\int_{M} S S^{-1} \Lambda_{m}^{*} \Lambda_{m} S^{-1} f d \mu \\
& =\int_{M} \Lambda_{m}^{*} \Lambda_{m} S^{-1} f d \mu=S S^{-1} f=f .
\end{aligned}
$$

Hence $\tilde{S}=S^{-1}$ and $\tilde{\Lambda}_{m} \tilde{S}^{-1}=\Lambda_{m} S^{-1} S=\Lambda_{m}$. In other words, $\left\{\Lambda_{m}: m \in M\right\}$ and $\left\{\tilde{\Lambda}_{m}: m \in M\right\}$ are dual $g$-frames with respect to each other.

Remark 2.2. We can always get a tight $g$-frame from any $g$-frame $\left\{\Lambda_{m}: m \in M\right\}$. In fact if we put $\left\{Q_{m}=\Lambda_{m} S^{1 / 2}: m \in M\right\}$, it is easy to check that $\left\{Q_{m}: m \in M\right\}$ is a tight $g$-frame with the frame bound 1 .

Lemma 2.3. Let $\left\{\Lambda_{m}: m \in M\right\}$ be a g-frame for $U$ with respect to $\left\{V_{m}: m \in M\right\}$ and $\tilde{\Lambda}_{m}=\Lambda_{m} S^{-1}$. For any $g_{m} \in V_{m}$ satisfying $f=\int_{M} \Lambda_{m}^{*} g_{m} d \mu$, we have

$$
\int_{M}\left\|g_{m}\right\|^{2} d \mu=\int_{M}\left\|\tilde{\Lambda}_{m} f\right\|^{2} d \mu+\int_{M}\left\|g_{m}-\tilde{\Lambda}_{m} f\right\|^{2} d \mu .
$$

Proof. It is easy to check that for every $f \in U$,

$$
\begin{aligned}
\int_{M}\left\|\tilde{\Lambda}_{m} f\right\|^{2} d \mu & =\int_{M}\left\langle\tilde{\Lambda}_{m} f, \Lambda_{m} S^{-1} f\right\rangle d \mu=\int_{M}\left\langle\Lambda_{m}^{*} \tilde{\Lambda}_{m} f, S^{-1} f\right\rangle d \mu \\
& =\int_{M}\left\langle\Lambda_{m}^{*} g_{m}, S^{-1} f\right\rangle d \mu=\int_{M}\left\langle g_{m}, \Lambda_{m} S^{-1} f\right\rangle d \mu \\
& =\int_{M}\left\langle g_{m}, \tilde{\Lambda}_{m} f\right\rangle d \mu,
\end{aligned}
$$

and the conclusion follows.

\section{Generalized Bessel nets, Riesz bases and orthonormal bases}

Similar to generalized frames, we can define generalized Bessel nets, Riesz bases, and orthonormal bases. 
Definition 3.1. Let $\Lambda_{m} \in \mathscr{B}\left(U, V_{m}\right)$, for $m \in M$.

(i) If the right hand inequality of (1-1) holds, then we say that $\left\{\Lambda_{m}: m \in M\right\}$ is a $g$-Bessel net for $U$ with respect to $\left\{V_{m}: m \in M\right\}$.

(ii) If $\left\{f: \Lambda_{m} f=0(m \in M)\right\}=\{0\}$ then we say that $\left\{\Lambda_{m}: m \in M\right\}$ is $g$-complete.

(iii) If $\left\{\Lambda_{m}: m \in M\right\}$ is $g$-complete and there are two positive constant $A$ and $B$ such that for any measurable subset $M_{1} \subset M$ of finite measure, and $g_{m} \in$ $V_{m}, m \in M_{1}$,

$$
A \int_{M_{1}}\left\|g_{m}\right\|^{2} d \mu \leq\left\|\int_{M_{1}} \Lambda_{m}^{*} d \mu\right\|^{2} \leq B \int_{M_{1}}\left\|g_{m}\right\|^{2} d \mu,
$$

then we say $\left\{\Lambda_{m}: m \in M\right\}$ is a $g$-Riesz bases for $U$ with respect to the set $\left\{V_{m}: m \in M\right\}$.

(iv) We say $\left\{\Lambda_{m}: m \in M\right\}$ is a $g$-orthonormal basis for $U$ with respect to the set $\left\{V_{m}: m \in M\right\}$ if it satisfies the following equalities:

$$
\begin{gathered}
\left\langle\Lambda_{m_{1}}^{*} g_{m_{1}}, \Lambda_{m_{2}}^{*} g_{m_{2}}\right\rangle=\delta_{m_{1} m_{2}}\left\langle g_{m_{1}}, g_{m_{2}}\right\rangle\left(m_{1}, m_{2} \in M, g_{m_{1}} \in V_{m_{1}}, g_{m_{2}} \in V_{m_{2}}\right) \\
\int_{M}\left\|\Lambda_{m} f\right\|^{2} d \mu(m)=\left\|f^{2}\right\|, \quad(f \in U) .
\end{gathered}
$$

Characterization of $\boldsymbol{g}$-frames, $\boldsymbol{g}$-Riesz bases and $\boldsymbol{g}$-orthonormal bases. Consider $\Lambda_{m} \in \mathscr{B}\left(U, V_{m}\right)$; we do not have other assumptions on $\Lambda_{m}$ at the moment. Suppose that $\left\{e_{m, n}, n \in N_{m}\right\}$ is an orthonormal basis for $V_{m}$, where $N_{m}$ is an index set of arbitrary cardinality. Then

$$
f \mapsto\left\langle\Lambda_{m} f, e_{m, n}\right\rangle
$$

defines a bounded linear functional on $U$, so we can find $u_{m, n} \in U$ such that

$$
\left\langle f, u_{m, n}\right\rangle=\left\langle\Lambda_{m} f, e_{m, n}\right\rangle
$$

hence

$$
\Lambda_{m} f=\sum_{n \in N_{m}}\left\langle f, u_{m, n}\right\rangle e_{m, n}
$$

Since $\sum_{n \in N_{m}}\left|\left\langle f, u_{m, n}\right\rangle\right|^{2}=\left\|\Lambda_{m} f\right\|^{2} \leq\left\|\Lambda_{m}\right\|^{2}\|f\|^{2}$, the family $\left\{u_{m, n}: n \in N_{m}\right\}$ is a Bessel net for $U$, and it follows that for any $f \in U$ and $g \in V_{m}$,

$$
\left\langle f, \Lambda_{m}^{*} g\right\rangle=\left\langle\Lambda_{m} f, g\right\rangle=\sum_{n \in N_{m}}\left\langle f, u_{m, n}\right\rangle\left\langle e_{m, n}, g\right\rangle=\left\langle f, \sum_{n \in N_{m}}\left\langle g, e_{m, n}\right\rangle u_{m, n}\right\rangle .
$$

Hence

$$
\Lambda_{m}^{*} g=\sum_{n \in N_{m}}\left\langle g, e_{m, n}\right\rangle u_{m, n} \quad\left(g \in V_{m}\right)
$$

In particular,

$$
u_{m, n}=\Lambda_{m}^{*} e_{m, n} \quad\left(m \in M, n \in N_{m}\right)
$$


We call $\left\{u_{m, n}: m \in M, n \in N_{m}\right\}$ the net induced by $\left\{\Lambda_{m}: m \in M\right\}$ with respect to $\left\{e_{m, n}: n \in N_{m}, m \in M\right\}$. With these representations for $\Lambda_{m}^{*}$ and $\Lambda_{m}$, we get characterizations of generalized frames, Riesz bases and orthonormal bases.

Theorem 3.2. Let $\Lambda_{m} \in \mathscr{B}\left(U, V_{m}\right)$ and $u_{m, n}$ be defined as in (3-3).

(i) $\left\{\Lambda_{m}: m \in M\right\}$ is a g-frame (alternatively a $g$-Bessel net, tight g-frame, $g$-Riesz basis, or g-orthonormal basis) for $U$ if and only if $\left\{u_{m, n}: m \in M, n \in N_{m}\right\}$ is a frame (Bessel net, tight frame, Riesz basis, orthonormal basis) for $U$.

(ii) If $\left\{\Lambda_{m}: m \in M\right\}$ is a $g$-frame, then

$$
\sum_{m \in M} \operatorname{dim} V_{m} \geq \operatorname{dim} U
$$

and the equality holds whenever $\left\{\Lambda_{m}: m \in M\right\}$ is a g-Riesz basis.

(iii) The $g$-frame operator for $\left\{\Lambda_{m}: m \in M\right\}$ coincides with the frame operator for $\left\{u_{m, n}: m \in M, n \in N_{m}\right\}$.

(iv) $\left\{\Lambda_{m}: m \in M\right\}$ and $\left\{\tilde{\Lambda}_{m}: m \in M\right\}$ are a pair of (canonical) dual g-frames if and only if the induced net are a pair of (canonical) dual frames.

Proof. (i) We see from (3-4) that

$$
\int_{M}\left\|\Lambda_{m} f\right\|^{2} d \mu(m)=\int_{M} \sum_{n \in N_{m}}\left|\left\langle f, u_{m, n}\right\rangle\right|^{2} d \mu(m), \quad(f \in U) .
$$

Hence $\left\{\Lambda_{m}: m \in M\right\}$ is a $g$-frame (respectively $g$-Bessel net, tight-frame) for $U$ if and only if $\left\{u_{m, n}: m \in M, n \in N_{m}\right\}$ is a frame (respectively Bessel net, tight frame) for $U$.

Next assume that $\left\{\Lambda_{m}: m \in M\right\}$ is a $g$-Riesz bases for $U$. Since $\left\{e_{m, n}: n \in N_{m}\right\}$ is an orthonormal basis for $V_{m}$, every $g_{m} \in V_{m}$ has an expansion of the form $g_{m}=$ $\sum_{n \in N_{m}} c_{m, n} e_{m, n}$, where $\left\{c_{m, n}: n \in N_{m}\right\} \in l^{2}\left(N_{m}\right)$. It follows that

$$
A \int_{M_{1}}\left\|g_{m}\right\|^{2} d \mu \leq\left\|\int_{M_{1}} \Lambda_{m}^{*} g_{m} d \mu\right\|^{2} \leq B \int_{M_{1}}\left\|g_{m}\right\|^{2} d \mu
$$

is equivalent to

$$
A \int_{M_{1}}\left(\sum_{n \in N_{m}}\left|c_{m, n}\right|^{2}\right) d \mu \leq\left\|\int_{M_{1}}\left(\sum_{n \in N_{m}} c_{m, n} u_{m, n}\right) d \mu\right\|^{2} \leq B \int_{M_{1}}\left(\sum_{n \in N_{m}}\left|c_{m, n}\right|^{2}\right) d \mu .
$$

On the other hand, we see from $\Lambda_{m} f=\sum_{n \in N_{m}}\left\langle f, u_{m, n}\right\rangle e_{m, n}$ that

$$
\left\{f: \Lambda_{m} f=0(m \in M)\right\}=\left\{f:\left\langle f, u_{m, n}\right\rangle=0\left(m \in M, n \in N_{m}\right)\right\} .
$$

Hence $\left\{\Lambda_{m}: m \in M\right\}$ is $g$-complete if and only if $\left\{u_{m, n}: m \in M, n \in N_{m}\right\}$ is complete. Therefor $\left\{\Lambda_{m}: m \in M\right\}$ is a $g$-Riesz basis if and only if $\left\{u_{m, n}: m \in M, n \in N_{m}\right\}$ is a Riesz basis. 
Now assume that $\left\{\Lambda_{m}: m \in M\right\}$ is a $g$-orthonormal basis. It follows from (3-1) and (3-3) that

$$
\begin{aligned}
\left\langle u_{m_{1}, n_{1}}, u_{m_{2}, n_{2}}\right\rangle & =\left\langle\Lambda_{m_{2}} u_{m_{1}, n_{1}}, e_{m_{2}, n_{2}}\right\rangle=\overline{\left\langle\Lambda_{m_{2}}^{*} e_{m_{2}, n_{2}}, u_{m_{1}, n_{1}}\right\rangle} \\
& =\overline{\left\langle\Lambda_{m_{1}} \Lambda_{m_{2}}^{*} e_{m_{2}, n_{2}}, e_{m_{1}, n_{1}}\right\rangle}=\left\langle\Lambda_{m_{1}}^{*} e_{m_{1}, n_{1}}, \Lambda_{m_{2}}^{*} e_{m_{2}, n_{2}}\right\rangle=\delta_{m_{1}, m_{2}} \delta_{n_{1}, n_{2}}
\end{aligned}
$$

for $m_{1}, m_{2} \in M, n_{1} \in N_{m_{1}}, n_{2} \in N_{m_{2}}$. Hence $\left\{u_{m, n}, m \in M, n \in N_{m}\right\}$ is an orthonormal net. Moreover, observe that

$$
\|f\|^{2}=\int_{M}\left\|\Lambda_{m} f\right\|^{2} d \mu=\int_{M}\left(\sum_{n \in N_{m}}\left|\left\langle f, u_{m, n}\right\rangle\right|\right) d \mu \quad(f \in U) .
$$

Therefore $\left\{u_{m, n}: m \in M, n \in N_{m}\right\}$ is an orthonormal basis.

For the converse, we need only to show that (3-2) holds. In fact, we see from (3-5) that for any $m_{1} \neq m_{2} \in M, g_{m_{1}} \in V_{m_{1}}, g_{m_{2}} \in V_{m_{2}}$,

$$
\left\langle\Lambda_{m_{1}}^{*} g_{m_{1}}, \Lambda_{m_{2}}^{*} g_{m_{2}}\right\rangle=\left\langle\sum_{n_{1} \in N_{m_{1}}}\left\langle g_{m_{1}}, e_{m_{1}, n_{1}}\right\rangle u_{m_{1}, n_{1}}, \sum_{n_{2} \in N_{m_{2}}}\left\langle g_{m_{2}}, e_{m_{2}, n_{2}}\right\rangle u_{m_{2}, n_{2}}\right\rangle=0
$$

and for $m \in M, g_{1}, g_{2} \in V_{m}$,

$$
\left\langle\Lambda_{m}^{*} g_{1}, \Lambda_{m}^{*} g_{2}\right\rangle=\left\langle\sum_{n_{1} \in N_{m}}\left\langle g_{1}, e_{m, n_{1}}\right\rangle u_{m, n_{1}}, \sum_{n_{2} \in N_{m}}\left\langle g_{2}, e_{m, n_{2}}\right\rangle u_{m, n_{2}}\right\rangle=\left\langle g_{1}, g_{2}\right\rangle .
$$

Now the conclusion follows.

(ii) Since the cardinality of a frame is no less than that of the basis, we have $\#\left\{u_{m, n}: m \in M, n \in N_{m}\right\} \geq \operatorname{dim} U$. Moreover, we see from (i) that the equality holds whenever $\left\{\Lambda_{m}: m \in M\right\}$ is a $g$-Riesz basis.

(iii) We see from (3-4) and (3-5) that, for $f \in U$,

$$
\begin{aligned}
\int_{M} \Lambda_{m}^{*} \Lambda_{m} f d \mu & =\int_{M}\left(\sum_{n \in N_{m}}\left\langle\Lambda_{m} f, e_{m, n}\right\rangle u_{m, n}\right) d \mu \\
& =\int_{M}\left(\sum_{n \in N_{m}}\left\langle\sum_{n^{\prime} \in N_{m}}\left\langle f, u_{m, n^{\prime}}\right\rangle e_{m, n^{\prime}}, e_{m, n}\right\rangle u_{m, n}\right) d \mu \\
& =\int_{M}\left(\sum_{n \in N_{m}}\left\langle f, u_{m, n}\right\rangle u_{m, n}\right) d \mu
\end{aligned}
$$

Hence the $g$-frame operator for $\left\{\Lambda_{m}: m \in M\right\}$ coincides with the frame operator for $\left\{u_{m, n}: m \in M, n \in N_{m}\right\}$.

(iv) This is the content of (i) and (iii). 
Corollary 3.3. $\left\{\Lambda_{m}: m \in M\right\}$ is a g-Bessel net with an upper bound $B$ if and only if for any measurable subset $M_{1} \subset M$ of finite measure,

$$
\left\|\int_{M_{1}} \Lambda_{m}^{*} d \mu\right\|^{2} \leq B \int_{M_{1}}\left\|g_{m}\right\|^{2} d \mu .
$$

Corollary 3.4. A g-Riesz basis $\left\{\Lambda_{m}: m \in M\right\}$ is an exact $g$-frame. Moreover, it is g-biorthonormal with respect to its dual $\left\{\tilde{\Lambda}_{m}: m \in M\right\}$ in the sense that

$$
\left\langle\Lambda_{m_{1}}^{*} g_{m_{1}}, \Lambda_{m_{2}}^{*} g_{m_{2}}\right\rangle=\delta_{m_{1}, m_{2}}\left\langle g_{m_{1}}, g_{m_{2}}\right\rangle \quad\left(m_{1}, m_{2} \in M, g_{m_{1}} \in V_{m_{1}}, g_{m_{2}} \in V_{m_{2}}\right) .
$$

Corollary 3.5. A net $\left\{\Lambda_{m}: m \in M\right\}$ is a g-Riesz basis for $U$ with respect to the set $\left\{V_{m}: m \in M\right\}$ if and only if there is a g-orthonormal basis $\left\{Q_{m}: m \in M\right\}$ for $U$ and a bounded invertible linear operator $T$ on $U$ such that $\Lambda_{m}=Q_{m} T, m \in M$.

Proof. Let $\left\{e_{m, n}: n \in N_{m}\right\}$ be an orthonormal basis for $V_{m}, m \in M$. First, we assume that $\left\{\Lambda_{m}: m \in M\right\}$ is a $g$-Riesz basis for $U$. By Theorem 3.2, we can find some Riesz basis $\left\{u_{m, n}: m \in M, n \in N_{m}\right\}$ for $U$ such that

$$
\Lambda_{m} f=\sum_{n \in N_{m}}\left\langle f, u_{m, n}\right\rangle e_{m, n}
$$

Take an orthonormal basis $\left\{u_{m, n}^{0}\right\}$ for $U$ and define the operator $T$ on $U$ by

$$
T^{*} u_{m, n}^{0}=u_{m, n} .
$$

Obviously, $T$ is a bounded invertible operator. Let $Q_{m} \in \mathscr{B}\left(U, V_{m}\right)$ be such that $Q_{m} f=\sum_{n \in N_{m}}\left\langle f, u_{m, n}^{0}\right\rangle e_{m, n}$. Again by Theorem 3.2, $\left\{Q_{m}: m \in M\right\}$ is a $g$ orthonormal basis for any $f \in U$, and

$$
\begin{aligned}
Q_{m} T f & =\sum_{n \in N_{m}}\left\langle T f, u_{m, n}^{0}\right\rangle e_{m, n}=\sum_{n \in N_{m}}\left\langle f, T^{*} u_{m, n}^{0}\right\rangle e_{m, n} \\
& =\sum_{n \in N_{m}}\left\langle T f, u_{m, n}\right\rangle e_{m, n}=\Lambda_{m} f .
\end{aligned}
$$

Hence $\Lambda_{m}=Q_{m} T$, for each $m \in M$.

Next we assume that $\left\{Q_{m}: m \in M\right\}$ is a $g$-orthonormal basis and $\Lambda_{m}=Q_{m} T$ for some bounded invertible operator $T$. Then $\left\{\Lambda_{m}: m \in M\right\}$ is $g$-complete in $U$ and we can find orthonormal basis $\left\{u_{m, n}^{0}: m \in M, n \in N_{m}\right\}$ for $U$ such that $Q_{m} f=\sum_{n \in N_{m}}\left\langle f, u_{m, n}^{0}\right\rangle e_{m, n}$. Hence

$$
\Lambda_{m} f=\sum_{n \in N_{m}}\left\langle T f, u_{m, n}^{0}\right\rangle e_{m, n}=\sum_{n \in N_{m}}\left\langle f, T^{*} u_{m, n}^{0}\right\rangle e_{m, n},
$$

and we see from Theorem 3.2 that $\left\{\Lambda_{m}: m \in M\right\}$ is a $g$-Riesz basis. 
Excess of $g$-frames. By Theorem 3.2, $g$-frames, $g$-Riesz basis and $g$-orthonormal bases have properties similar to those of frame, Riesz bases and orthonormal bases, respectively. However, not all the properties are similar. For example, Riesz bases are equivalent to exact frames, but this is not the case for $g$-Riesz bases and exact $g$-frames.

In fact, we see from Theorem 3.2 that a $g$-Riesz basis is also a $g$-frame, while the converse is not true. This is not surprising, since one element of a $g$-frame might correspond to several elements of the induced frame.

A natural problem arises: given a measurable subset $M_{1} \subset M$ with $\mu\left(M_{1}\right)>0$, when is $\left\{\Lambda_{m}: m \in M-M_{1}\right\}$ a $g$-frame?

Theorem 3.6. Let $\left\{\Lambda_{m}: m \in M\right\}$ be a g-frame for $U$ with respect to $\left\{V_{m}: m \in M\right\}$ and $\left\{\tilde{\Lambda}_{m}: m \in M\right\}$ be the canonical dual $g$-frame. Suppose that $M_{1} \subset M$ and $\mu\left(M_{1}\right)>0$.

(i) If there is some $g_{0} \in V_{m^{\prime}}-\{0\}$ such that $\tilde{\Lambda}_{m^{\prime}} \Lambda_{m^{\prime}}^{*} g_{0}=g_{0}$, for any $m^{\prime} \in M_{1}$, then $\left\{\Lambda_{m}: m \in M-M_{1}\right\}$ is not $g$-complete in $U$.

(ii) If there is some $f_{0} \in U-\{0\}$ such that $\Lambda_{m^{\prime}}^{*} \tilde{\Lambda}_{m^{\prime}} f_{0}=f_{0}$, for any $m^{\prime} \in M_{1}$, then $\left\{\Lambda_{m}: m \in M-M_{1}\right\}$ is not $g$-complete in $U$.

(iii) If $I-\Lambda_{m^{\prime}}^{*} \tilde{\Lambda}_{m^{\prime}}^{*}$ or $I-\tilde{\Lambda}_{m^{\prime}} \Lambda_{m^{\prime}}^{*}$ is bounded invertible on $V_{m^{\prime}}$, for any $m^{\prime} \in M_{1}$, then $\left\{\Lambda_{m}: m \in M-M\right\}$ is a $g$-frame for $U$.

Proof. (i) Since $\Lambda_{m^{\prime}}^{*} g_{0} \in U$, we have from (2-2)

$$
\Lambda_{m^{\prime}}^{*} g_{0}=\int_{M} \Lambda_{m}^{*} \tilde{\Lambda}_{m} \Lambda_{m^{\prime}}^{*} g_{0} d \mu
$$

Put $v_{m^{\prime}, m}=\delta_{m^{\prime}, m} g_{0}$. We have

$$
\Lambda_{m^{\prime}}^{*} g_{0}=\int_{M} \Lambda_{m}^{*} v_{m^{\prime}, m} d \mu .
$$

It follows from Lemma 2.3 that

$$
\int_{M}\left\|v_{m_{0}, m}\right\|^{2} d \mu=\int_{M}\left\|\tilde{\Lambda}_{m} \Lambda_{m_{0}}^{*} g_{0}\right\|^{2} d \mu+\int_{M}\left\|\tilde{\Lambda}_{m} \Lambda_{m_{0}}^{*} g_{0}-v_{m_{0}, m}\right\|^{2},
$$

and so

$$
\left\|g_{0}\right\|^{2}=\left\|g_{0}\right\|^{2}+2 \int_{M-M_{1}}\left\|\tilde{\Lambda}_{m} \Lambda_{m^{\prime}}^{*} g_{0}\right\|^{2} d \mu .
$$

Hence $\tilde{\Lambda}_{m} \Lambda_{m^{\prime}}^{*} g_{0}=0$, for each $m^{\prime} \in M_{1}$ and almost all $m \in M-M_{1}$. This means that $\Lambda_{m} \tilde{\Lambda}_{m^{\prime}}^{*} g_{0}=\Lambda_{m} S^{-1} \Lambda_{m^{\prime}}^{*} g_{0}=\tilde{\Lambda}_{m} \Lambda_{m^{\prime}}^{*} g_{0}=0$, for almost all $m \neq m^{\prime}$. But $\left\langle\Lambda_{m^{\prime}}^{*} g_{0}, \tilde{\Lambda}_{m^{\prime}}^{*} g_{0}\right\rangle=\left\langle\tilde{\Lambda}_{m^{\prime}} \Lambda_{m^{\prime}}^{*} g_{0}, g_{0}\right\rangle=\left\|g_{0}\right\|^{2}>0$, which implies that $\tilde{\Lambda}_{m^{\prime}}^{*} g_{0} \neq 0$. Hence $\left\{\Lambda_{m}: m \in M-M_{1}\right\}$ is not $g$-complete in $U$. 
(ii) Since $\Lambda_{m^{\prime}}^{*} \tilde{\Lambda}_{m^{\prime}} f_{0}=f_{0} \neq 0$, we have $\tilde{\Lambda}_{m^{\prime}} f_{0} \neq 0$ and $\tilde{\Lambda}_{m^{\prime}} \Lambda_{m^{\prime}}^{*} f_{0}=\tilde{\Lambda}_{m^{\prime}} f_{0}$. Now the conclusion follows from (i).

(iii) Since $\tilde{\Lambda}_{m}=\Lambda_{m} S^{-1}$ where $S$ is the $g$-frame operator for $\left\{\Lambda_{m}: m \in M\right\}$, we have

$$
I-\Lambda_{m^{\prime}} \tilde{\Lambda}_{m^{\prime}}=I-\Lambda_{m^{\prime}} S^{-1} \tilde{\Lambda}_{m^{\prime}}^{*}=I-\tilde{\Lambda}_{m^{\prime}} \Lambda_{m^{\prime}}^{*}
$$

Let $A$ and $B$ be the lower and upper frame bounds for $\left\{\Lambda_{m}: m \in M\right\}$. For any $f \in U$, we have

$$
f=\int_{M} \tilde{\Lambda}_{m}^{*} \Lambda_{m} f d \mu
$$

Hence, for each $m^{\prime} \in M_{1}$,

$$
\Lambda_{m^{\prime}} f=\int_{M} \Lambda_{m^{\prime}} \tilde{\Lambda}_{m}^{*} \Lambda_{m} f d \mu
$$

Therefore

$$
\left(I-\Lambda_{m^{\prime}} \tilde{\Lambda}_{m^{\prime}}^{*}\right) \Lambda_{m^{\prime}} f=\int_{M-M_{1}} \Lambda_{m^{\prime}} \tilde{\Lambda}_{m}^{*} \Lambda_{m} f d \mu
$$

Note that

$$
\begin{aligned}
\left\|\int_{M-M_{1}} \Lambda_{m^{\prime}} \tilde{\Lambda}_{m}^{*} \Lambda_{m} f d \mu\right\|^{2} & \left.=\sup _{g \in V_{m^{\prime},\|g\|=1}} \| \int_{M-M_{1}} \Lambda_{m^{\prime}} \tilde{\Lambda}_{m}^{*} \Lambda_{m} f d \mu, g\right\rangle\left.\right|^{2} \\
& =\sup _{\|g\|=1}\left|\int_{M-M_{1}}\left\langle\Lambda_{m} f, \tilde{\Lambda}_{m} \Lambda_{m^{\prime}}^{*} g\right\rangle d \mu\right|^{2} \\
& \leq \int_{M-M_{1}}\left\|\Lambda_{m} f\right\|^{2} d \mu \sup _{\|g\|=1} \int_{M}\left\|\tilde{\Lambda}_{m} \Lambda_{m^{\prime}}^{*} g\right\|^{2} d \mu \\
& \leq \frac{1}{A}\left\|\Lambda_{m^{\prime}}\right\|^{2} \int_{M-M_{1}}\left\|\Lambda_{m} f\right\|^{2} d \mu .
\end{aligned}
$$

We see from (3-7) that

$$
\left\|\Lambda_{m^{\prime}} f\right\|^{2} \leq\left\|\left(I-\Lambda_{m^{\prime}} \tilde{\Lambda}_{m^{\prime}}^{*}\right)^{-1}\right\| \frac{1}{A}\left\|\Lambda_{m^{\prime}}\right\|^{2} \int_{M-M_{1}}\left\|\Lambda_{m} f\right\|^{2} d \mu \quad\left(m^{\prime} \in M_{1}\right) .
$$

Hence

$$
\int_{M}\left\|\Lambda_{m} f\right\|^{2} d \mu \leq C \int_{M-M_{1}}\left\|\Lambda_{m} f\right\|^{2} d \mu
$$

Therefore, for $f \in U$,

$$
\frac{A}{C}\|f\|^{2} \leq \int_{M-M_{1}}\left\|\Lambda_{m} f\right\|^{2} d \mu \leq B\|f\|^{2} .
$$

This completes the proof. 


\section{Applications of $g$-frames}

Atomic resolution of bounded linear operators. Here we give an application of $g$-frames. Let $\left\{\Lambda_{m}: m \in M\right\}$ be a $g$-frame for $U$ with respect to $\left\{V_{m}: m \in M\right\}$. Suppose that $\left\{\tilde{\Lambda}_{m}: m \in M\right\}$ is the canonical dual $g$-frame. Then for any $f \in U$, we have $f=\int_{M} \Lambda_{m}^{*} \tilde{\Lambda}_{m} f d \mu=\int_{M} \tilde{\Lambda}_{m}^{*} \Lambda_{m} f d \mu$. It follows that

$$
I_{U}=\int_{M} \Lambda_{m}^{*} \tilde{\Lambda}_{m} d \mu=\int_{M} \tilde{\Lambda}_{m}^{*} \Lambda_{m} d \mu .
$$

Let $T$ be a bounded linear operator on $U$. We see from (4-1) that

$$
T=\int_{M} T \Lambda_{m}^{*} \tilde{\Lambda}_{m} d \mu=\int_{M} T \tilde{\Lambda}_{m}^{*} \Lambda_{m} d \mu=\int_{M} \Lambda_{m}^{*} \tilde{\Lambda}_{m} T d \mu=\int_{M} \tilde{\Lambda}_{m}^{*} \Lambda_{m} T d \mu .
$$

Construction of frames via $g$-frames. Let $\left\{\Lambda_{m}: m \in M\right\}$ be a $g$-frame for $U$ with respect to $\left\{V_{m}: m \in M\right\}$. We see from Theorem 3.2 that

$$
\left\{u_{m, n}: m \in M, n \in N_{m}\right\}=\left\{\Lambda_{m}^{*} e_{m, n}: m \in M, n \in N_{m}\right\}
$$

is a frame for $U$, where $\left\{e_{m, n}: n \in N_{m}\right\}$ is an orthonormal basis for $V_{m}$. However, it might be difficult to find an orthonormal basis for $V_{m}$ in practice. Fortunately, orthonormality is not necessary to get a frame. In fact:

Theorem 4.1. Let $\left\{\Lambda_{m}: m \in M\right\}$ and $\left\{\tilde{\Lambda}_{m}: m \in M\right\}$ be a pair of dual $g$-frames for $U$ with respect to $\left\{V_{m}: m \in M\right\}$, and $\left\{g_{m, n}: n \in N_{m}\right\}$ and $\left\{\tilde{g}_{m, n}: n \in N_{m}\right\}$ be the corresponding pair of dual frames for $V_{m}$, respectively. Then

$$
\left\{\Lambda_{m}^{*} g_{m, n}: m \in M, n \in N_{m}\right\} \quad \text { and } \quad\left\{\tilde{\Lambda}_{m} \tilde{g}_{m, n}: m \in M, n \in N_{m}\right\}
$$

are a pair of dual frames for $U$, provided that the frame bounds for $\left\{g_{m, n}: n \in N_{m}\right\}$ satisfy $C_{1} \leq A_{m} \leq B_{m} \leq C_{2}$, for some constants $C_{1}, C_{2}>0$.

Moreover, suppose that $\left\{\Lambda_{m}: m \in M\right\}$ and $\left\{\tilde{\Lambda}_{m}: m \in M\right\}$ are canonical dual $g$-frames for $U,\left\{g_{m, n}: n \in N_{m}\right\}$ and $\left\{\tilde{g}_{m, n}: n \in N_{m}\right\}$ are canonical dual frames for $V_{m}$, and $\left\{g_{m, n}: n \in N_{m}\right\}$ is a tight $g$-frame with frame bounds $A_{m}=B_{m}=A$, $m \in M$. Then $\left\{\Lambda_{m}^{*} g_{m, n}: m \in M, n \in N_{m}\right\}$ and $\left\{\tilde{\Lambda}_{m}^{*} \tilde{g}_{m, n}: m \in M, n \in N_{m}\right\}$ are canonical dual frames for $U$.

Proof. Note that $\left\langle f, \Lambda_{m}^{*} g_{m, n}\right\rangle=\left\langle\Lambda_{m} f, g_{m, n}\right\rangle$. It is easy to see that $\left\{\Lambda_{m}^{*} g_{m, n}\right.$ : $\left.m \in M, n \in N_{m}\right\}$ and $\left\{\tilde{\Lambda}_{m}^{*} \tilde{g}_{m, n}: m \in M, n \in N_{m}\right\}$ are frames for $U$. On the other hand, for any $f \in U$, we have

$$
\begin{aligned}
\int_{M}\left(\sum_{n \in N_{m}}\left\langle f, \Lambda_{m}^{*} g_{m, n}\right\rangle \tilde{\Lambda}_{m}^{*} \tilde{g}_{m, n}\right) d \mu & =\int_{M} \tilde{\Lambda}_{m}^{*} \sum_{n \in N_{m}}\left\langle\Lambda_{m} f, g_{m, n}\right\rangle \tilde{g}_{m, n} d \mu \\
& =\int_{M} \tilde{\Lambda}_{m}^{*} \Lambda_{m} f=f .
\end{aligned}
$$


Similarly we can get

$$
\int_{M}\left(\sum_{n \in N_{m}}\left\langle f, \tilde{\Lambda}_{m}^{*} \tilde{g}_{m, n}\right\rangle \Lambda_{m}^{*} g_{m, n}\right) d \mu=f .
$$

Hence $\left\{\Lambda_{m}^{*} g_{m, n}: m \in M, n \in N_{m}\right\}$ and $\left\{\tilde{\Lambda}_{m}^{*} \tilde{g}_{m, n}: m \in M, n \in N_{m}\right\}$ are dual frames for $U$.

Next we assume that $\left\{\Lambda_{m}: m \in M\right\}$ and $\left\{\tilde{\Lambda}_{m}: m \in M\right\}$ are canonical dual $g$ frames and $\left\{g_{m, n}: n \in N_{m}\right\}$ is a tight frame with frames bounds $A_{m}=B_{m}=A$. Then $\tilde{g}_{m, n}=A^{-1} g_{m, n}$. Let $S_{\Lambda}$ and $S_{\Lambda, g}$ be the frame operators associated with $\left\{\Lambda_{m}: m \in M\right\}$ and $\left\{\Lambda_{m}^{*} g_{m, n}: m \in M, n \in N_{m}\right\}$, respectively. Then, for $f \in U$,

$$
\begin{aligned}
S_{\Lambda, g} f & =\int_{M}\left(\sum_{n \in N_{m}}\left\langle f, \Lambda_{m}^{*} g_{m, n}\right\rangle \Lambda_{m}^{*} g_{m, n}\right) d \mu \\
& =\int_{M}\left(\Lambda_{m}^{*} \sum_{n \in N_{m}}\left\langle\Lambda_{m} f, g_{m, n}\right\rangle g_{m, n}\right) d \mu=A \int_{M} \Lambda_{m}^{*} \Lambda_{m} f d \mu=A S_{\Lambda} f .
\end{aligned}
$$

Hence

$$
S_{\Lambda, g}^{-1} \Lambda_{m}^{*} g_{m, n}=\frac{1}{A} S_{\Lambda}^{-1} \Lambda_{m}^{*} g_{m, n}=\Lambda_{m}^{*} \tilde{g}_{m, n} \quad\left(m \in M, n \in N_{m}\right) .
$$

This completes the proof.

\section{References}

[Aldroubi et al. 2004] A. Aldroubi, C. Cabrelli, and U. M. Molter, "Wavelets on irregular grids with arbitrary dilation matrices and frame atoms for $L^{2}\left(\mathbb{R}^{d}\right)$ ", Appl. Comput. Harmon. Anal. 17:2 (2004), 119-140. MR 2006f:42035 Zbl 1060.42025

[Asgari and Khosravi 2005] M. S. Asgari and A. Khosravi, "Frames and bases of subspaces in Hilbert spaces”, J. Math. Anal. Appl. 308:2 (2005), 541-553. MR 2006b:42042 Zbl 1091.46006

[Casazza and Kutyniok 2004] P. G. Casazza and G. Kutyniok, "Frames of subspaces", pp. 87-113 in Wavelets, frames and operator theory, edited by C. Heil et al., Contemp. Math. 345, Amer. Math. Soc., Providence, RI, 2004. MR 2005e:42090 Zbl 1058.42019

[Christensen 2003] O. Christensen, An introduction to frames and Riesz bases, Birkhäuser, Boston, 2003. MR 2003k:42001 Zbl 1017.42022

[Christensen and Eldar 2004] O. Christensen and Y. C. Eldar, "Oblique dual frames and shiftinvariant spaces”, Appl. Comput. Harmon. Anal. 17:1 (2004), 48-68. MR 2005f:42065 Zbl 1043. 42027

[Daubechies 1992] I. Daubechies, Ten lectures on wavelets, CBMS-NSF Regional Conference Series in Applied Mathematics 61, Society for Industrial and Applied Mathematics (SIAM), Philadelphia, PA, 1992. MR 93e:42045 Zbl 0776.42018

[Daubechies et al. 1986] I. Daubechies, A. Grossmann, and Y. Meyer, "Painless nonorthogonal expansions”, J. Math. Phys. 27:5 (1986), 1271-1283. MR 87e:81089 Zbl 0608.46014

[Duffin and Schaeffer 1952] R. J. Duffin and A. C. Schaeffer, "A class of nonharmonic Fourier series”, Trans. Amer. Math. Soc. 72 (1952), 341-366. MR 13,839a Zbl 0049.32401 
[Feichtinger and Strohmer 1998] H. G. Feichtinger and T. Strohmer (editors), Gabor analysis and algorithms: Theory and applications, Birkhäuser, Boston, 1998. MR 98h:42001

[Fornasier 2003] M. Fornasier, "Decompositions of Hilbert spaces: local construction of global frames", pp. 275-281 in Constructive theory of functions, edited by B. Bojanov, DARBA, Sofia, 2003. MR 2005f:42085 Zbl 1031.42035

[Gröchenig 2001] K. Gröchenig, Foundations of time-frequency analysis, Birkhäuser, Boston, 2001. MR 2002h:42001 Zbl 0966.42020

[Han and Larson 2000] D. Han and D. R. Larson, "Frames, bases and group representations", Mem. Amer. Math. Soc. 147:697 (2000), x+94. MR 2001a:47013

[Heil and Walnut 1989] C. E. Heil and D. F. Walnut, "Continuous and discrete wavelet transforms", SIAM Rev. 31:4 (1989), 628-666. MR 91c:42032 Zbl 0683.42031

[Sun 2006] W. Sun, "G-frames and g-Riesz bases", J. Math. Anal. Appl. 322:1 (2006), 437-452. MR 2007b:42047 Zbl 1129.42017

[Yong 1980] R. Yong, An Introduction to non-harmonic Fourier series, Academic Press, New York, 1980.

Received: 2008-09-29 Accepted: 2009-03-19

reza_joveini@bojnourdiau.ac.ir Faculty of Science, Islamic Azad University of Bojnourd, Bojnourd 94176-94686, Iran

mamini@modraes.ac.ir

Faculty of Mathematical Sciences,

Tarbiat Modares University, Tehran 14115-175, Iran

Current address:

Institut Penyelidikan Matematik, Universiti Putra Malaysia, 43400 UPM Serdang, Selangor Darul Ehsan, Malaysia 